\title{
Idiopathic orbital inflammation (orbital inflammatory pseudotumour): an unusual cause of transient ischaemic attack
}

\author{
F-X Borruat, P Vuilleumier, N Ducrey, H Fankhauser, R C Janzer, F Regli
}

\begin{abstract}
A patient with idiopathic inflammation of the right orbit, established by biopsy, developed episodes of transient left sensorimotor hemiparesis. Neuroimaging showed intracranial extension of the disease with pronounced narrowing of the right internal carotid artery in its intracavernous portion. Oral cyclophosphamide induced gradual improvement. Transient ischaemic attack is rarely found in association with orbital disease and indicates possible intracranial extension.
\end{abstract}

$(F$ Neurol Neurosurg Psychiatry 1995;58:88-90)

Keywords: idiopathic orbital inflammation; ischaemic attack; magnetic resonance imaging

Idiopathic orbital inflammation, previously known as orbital inflammatory pseudotumour, is a non-specific idiopathic inflammatory process that can involve any tissue of the orbit. Painful exophthalmos is a hallmark of the disease, as is its response to corticosteroid treatment. Visual loss is the major complication and may be related to optic nerve compression, retinal detachment, intraocular inflammation, glaucoma, or retinal vascular occlusion. ${ }^{1}$ Some patients with idiopathic orbital inflammation exhibit signs of meningeal irritation with pleocytosis and raised protein concentration in CSF. ${ }^{1}$ Bony erosion and intracranial extension are very rare complications ${ }^{2-10}$ and their occurrence should always lead to further investigation, including biopsy, to rule out malignancies or other aggressive orbital diseases.

We treated a patient for idiopathic inflammation of the right orbit who suddenly presented with transient left hemiparesis and hemiparaesthesia as the first sign of intracranial extension of the disease, eight months after the onset of orbital disease.

\section{Case report}

In June 1993 a 33 year old man was admitted for episodes of transient left hemiparesis and hemiparaesthesia.

Eight months earlier he had right painful exophthalmos, diplopia, and visual loss.
Computerised tomography showed right ethmoiditis, first treated with oral antibiotics and steroids, then by ethmoidectomy. Partial improvement occurred with oral antibiotics and steroids: visual acuity recovered fully, diplopia disappeared, but right orbital pain remained unchanged. In December 1992 diplopia and right visual loss increased and repeat CT showed enlargement of the posterior half of the left lateral rectus muscle; there was no evidence of disease elsewhere in the left orbit or cavernous sinus. Idiopathic orbital inflammation was suspected and with oral doses of prednisone ( $80-40 \mathrm{mg} /$ day), the course was fluctuating with persistent orbital pain.

In February 1993 visual acuity suddenly dropped to $20 / 200$ in the right eye. Magnetic resonance imaging showed massive enlargement of the posterior half of the lateral and superior rectus muscles on the right side, compressing the optic nerve at the apex; both cavernous sinuses were normal. Blood tests and CSF examination were unremarkable: erythrocyte sedimentation rate was $15 \mathrm{~mm}$; autoantibody screening, including cANCA and pANCA, was negative; immunofixation and HIV serology were negative; serum $\beta_{2}$ microglobulin and angiotensin converting enzyme were normal, as well as chest radiographs and whole body CT.

Frontal craniotomy was performed and the right optic canal was decompressed. Orbital biopsy showed mixed inflammatory infiltration including lymphocytes, plasmocytes, histiocytes, and some epithelioid cells; rare Langerhans type multinucleated giant cells were present and occasional areas of necrosis were noted. Immunohistochemical staining showed numerous macrophages and mostly $T$ lymphocytes without a monoclonal pattern. Pathological diagnosis was partly necrotising granulomatous soft tissue inflammation. Despite negative examination and cultures for any infective agent, the patient was treated with a combination of rifampicin-ethambutolisoniazid, due to a history of tuberculous pleuritis in 1987. Oral prednisone (60 mg/day) was continued. In April 1993, right eye visual acuity was 20/40; there was a $2 \mathrm{~mm}$ exophthalmos and diplopia was absent, but right orbital pain was still present.

In early June 1993, the patient experienced eight episodes of transient left hemiparesis 
Figure 1 Magnetic resonance imaging, $T 1$ weighted sequence $(T R=605, T E=15)$ after gadolinium injection. The right orbit is filled with an ill defined mass enhancing with gadolinium. Posterior scleritis is present (thickened posterior sclera with subsequent flattening of the globe) (white arrow). There is invasion of the right cavernous sinus and posterior extension along the edge of the cerebellar tentorium (small black arrows). The intracavernous portion of the internal carotid artery is barely visible large black arrow).

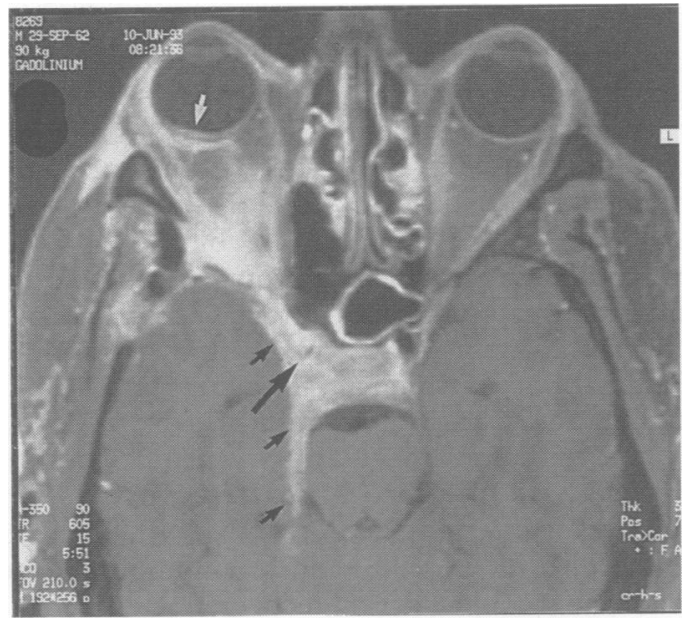

and hemisensory disturbances. Most of them were purely sensitive, lasting three to six minutes. They usually started in the left hand, progressively involving the whole arm over one to two minutes, clearing totally one to two minutes later. Twice, left facial numbness with dysarthria also occurred, but only once (the last episode) did he experience the same symptoms in the left leg together with weakness of his left side. Extracranial neurological examination was normal. An EEG and electroencephalographic continuous monitoring showed no epileptiform abnormalities. On 6 June 1993, neuro-ophthalmological examination showed the following: right eye visual acuity was $20 / 200$ without correction, improvable to $20 / 40$ with $a+4.5$ diopter lens; colour vision was $0 / 17$ on Ishihara plates with a right afferent pupillary defect; a $2 \mathrm{~mm}$ right exophthalmos was present and, except for partial adduction (60\%), ocular movements of the right eye were absent with a positive forced duction test and pronounced folds were visible on fundus examination; left eye vision was $20 / 20$ without correction and colour vision was $14 / 17$. Further extension of the orbital process was shown by MRI: firstly, the previously involved extraocular muscles were more enlarged; secondly, the posterior part of the globe was thickened with flattening of the globe, accounting for the induced hyperopia and retinal folds; and thirdly, most

Figure 2 Magnetic resonance angiography $(T R=33, T E=8)$ showing pronounced narrowing of the right intracavernous portion of the internal carotid artery (white arrow). resistance to retropulsion; prominent retinal

impressively, the process extended intracranially through the superior orbital fissure into the right cavernous sinus and along the right tentorial edge (fig 1). Magnetic resonance angiography (MRA) delineated the severe narrowing of the intracavernous portion of the right internal carotid artery (fig 2).

Frontal craniotomy was again performed. The right cavernous sinus was fibrosed and no venous haemorrhage occurred on incision; the right orbital apex was packed with fibrous tissue. Extensive biopsy was performed. Examination by microscopy showed dense fibrous tissue infiltrating striate muscles, mixed inflammatory cells (mainly $\mathrm{T}$ cell lymphocytes), numerous macrophages, numerous multinucleated giant cells, some plasmocytes, and epithelioid cells, with rare foci of necrosis, compatible with granulomatous inflammatory lesion. Molecular biology and immunostaining studies failed to show a monoclonal pattern. Bacterial, mycobacterial, and fungal cultures were all negative. Again, extensive biological investigations were negative for systemic inflammatory or granulomatous disorders, vasculitides, or malignancies. Tests of humoral and cell mediated immunity were normal.

The diagnosis of idiopathic orbital inflammation was established and oral cyclophosphamide (100 mg per day) was added to the previous treatment (prednisone $(40 \mathrm{mg}$ ) and rifampicin $(600 \mathrm{mg})$ daily). Gradual and slow improvement of vision, oculomotility, exophthalmos, and pain ensued. In December 1993 prednisone and rifampicin were discontinued and the patient was given $100 \mathrm{mg}$ cyclophosphamide per day only.

Evolution was favourable and in January 1994 no more abnormal tissue was found on MRI with gadolinium injection. Repeat MRA showed no improvement of flow within the right internal carotid artery. On 25 April 1994, the patient was still taking $100 \mathrm{mg}$ cyclophosphamide daily. Right eye visual acuity was 20/40, colour vision was $5 / 17$ (Ishihara), optic atrophy was present, and the Goldmann visual field was moderately concentrically narrowed. Enophthalmos of the right eye was then present $(4 \mathrm{~mm})$. Oculomotility had partially recovered (abduction $80 \%$, adduction $80 \%$, supraduction $60 \%$, infraduction $0 \%$ ) with a positive forced duction test. The left eye was normal. Transient ischaemic attacks had not recurred.

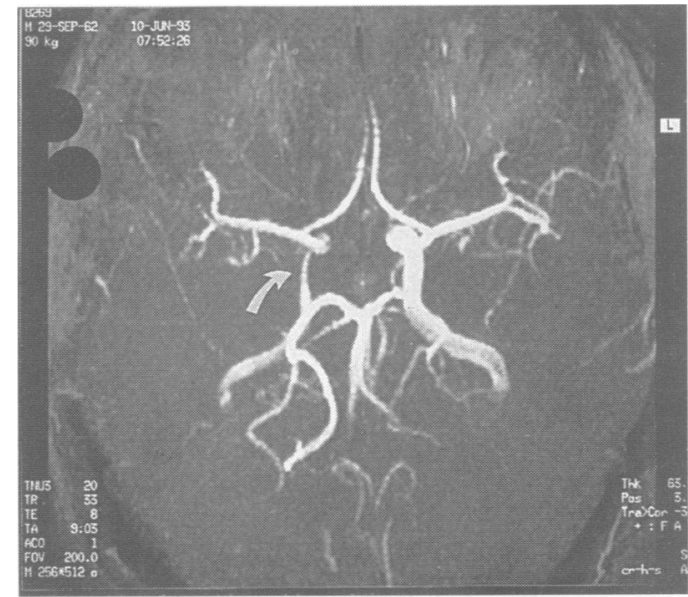

Eight months after the onset of the disease, intracranial extension of idiopathic orbital inflammation was heralded by transient left hemiparesis and hemiparaesthesia. MRI revealed extension of idiopathic orbital inflammation in the orbit with, firstly, myositis and posterior scleritis and, secondly, spreading through the superior orbital fissure and optic canal. Furthermore, left cavernous sinus invasion, extension along the cerebellar tentorial edge towards the posterior fossa, and severe internal carotid artery stenosis

\section{Discussion}


were found on MRI and MRA (figs 1 and 2).

Intracranial extension of an orbital process thought to be idiopathic orbital inflammation should raise suspicion and the diagnosis should be reconsidered unless proved by extensive biopsy. ${ }^{2}$ We obtained two extensive biopsies from our patient, the first before neuroradiological evidence of intracranial extension and the second when intracranial extension was present, each consistent with idiopathic orbital inflammation. Orbital exploration or biopsy of such cases are well known to exacerbate the inflammatory process and such manoeuvres should be performed only when the diagnosis is questioned by the unusual course of the disease. Indeed in our case intracranial extension could have been promoted by both biopsy and optic canal unroofing, leaving an opening to the endocranium. Such an intracranial extension, however, has been described without previous biopsy or decompressive procedure..$^{34910}$

Eighteen cases of extraorbital extension of idiopathic orbital inflammation have been reported to date, proved by biopsy in 10 of them. ${ }^{3-10}$ Erosion of bone was present in eight cases; extension through the superior orbital fissure in 11 , through an inferior orbital fissure in two, and through the optic canal in one. The cavernous sinus was invaded in nine patients and thick enhancing meninges were detected in four patients. Middle cranial fossa was invaded in 10 cases; posterior, infratemporal, and pterygopalatine fossa in one case each. A large intracranial mass was found in one case. Despite extensive disease, neurological signs of intracranial extension were scarce: one case of seizure, ${ }^{3}$ one case of hydrocephalus-dementia-ataxia, ${ }^{5}$ and one case of transient hemiparesis. ${ }^{7}$ Mechanisms for the last complication were not specified. In our patient, MRI and MRA showed tight narrowing of the right intracavernous internal carotid artery due to extension of disease, which is consistent with a history of repeated transient ischaemic attacks in the territory of the middle cerebral artery.

High dose oral corticosteroids are usually the treatment of choice in idiopathic orbital inflammation. Before the era of modern neuroimaging techniques, it was even considered as a therapeutic test. Our patient failed to respond to therapeutic doses of steroids and only partial improvement was achieved with doses of up to $1.5 \mathrm{mg} / \mathrm{kg}$ daily. Radiotherapy was considered, but results from biopsy showed a preponderance of fibrotic tissue and this treatment was discarded. As inflammatory cells were mostly $\mathrm{T}$ cell lymphocytes, oral cyclophosphamide was started in July 1993. With a $100 \mathrm{mg}$ daily dose of cyclophosphamide, gradual improvement ensued over the next seven months. Neurological symptoms never recurred. Pain and exophthalmos completely disappeared and oculomotility and visual acuity partially recovered.

This case emphasises the variety of symptoms and the aggressive behaviour that can be encountered with idiopathic orbital inflammation. To our knowledge, transient ischaemic attacks with hemiparesis have been reported only once apart from the present case.

We thank Dr WR Lee of Glasgow, UK and Dr J-P Deruaz and Dr F Delacrètaz of Lausanne, Switzerland for helpful comments on the pathology studies.

1 Jakobiec FA, Jones IS. Orbital inflammations. In: Tasman W, Jaeger EA, eds. Duane's clinical ophthalmology. Vol 2. Philadelphia: JB Lippincott Co, 1992.

2 Friedman DI, Feldon SE, Hudson HL, Hinton DR. Painful ophthalmoplegia - much more than meets the eye. Presented at the Frank B Walsh Society 23rd annual meetPresented at the Frank B Walsh Society 23rd an

3 Jackson $H$. Pseudotumour of the orbit. Br $₹$ Ophthalmol 1958;42:212-24.

4 Edwards MK, Zauel DW, Gilmor RL, Muller J. Invasive orbital pseudotumor-CT demonstration of extension beyond orbit. Neuroradiology 1982;23:215-7.

5 Kaye AH, Hahn JF, Craciun A, Hanson M, Berlin AJ, Tubbs RR. Intracranial extension of inflammatory pseudotumor of the orbit. $\mathcal{F}$ Neurosurg 1984;60:625-9.

6 Noble SC, Chandler WF, Lloyd RV. Intracranial extension of orbital pseudotumor: a case report. Neurosurgery 1986;18:798-801.

7 Frohman LP, Kupersmith MJ, Lang J, Reede D, Bergeron $\mathrm{RT}$, Aleksic S, Trasi S. Intracranial extension and bone destruction in orbital pseudotumor. Arch Ophthalmol 1986;104:380-4.

8 Clifton AG, Borgstein RL, Moseley IF, Kendall BE, Shaw PJ. Intracranial extension of orbital pseudotumour. Clin J. Intracranial extensi

9 Bencherif B, Zouaoul A, Chedid G, Kujas M, Van Effenterre $R$, Marsault C. Intracranial extension of an Effenterre $\mathrm{R}$, Marsault $\mathrm{C}$. Intracranial extension of an idiopathic orbital inflammatory

10 Diamond JP, Bloom PA, Ragge N, Easty DL, Laszlo G. Localized Wegener's granulomatosis presenting as an orbital pseudotumour with extension into the posterior cranial fossa. Eur $\mathcal{F}$ Ophthalmol 1993;3:143-6. 